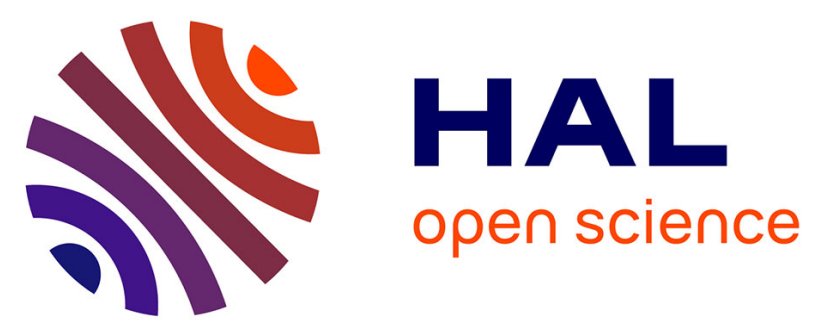

\title{
Fms-like Tyrosine Kinase 3 Ligand Concentration Kinetic Profile during Induction Is Strongly Predictive of Survivals in AML: Results of the FLAM/Flal Study
}

Pierre Peterlin, Joëlle Gaschet, Thierry Guillaume, Alice Garnier, Marion Eveillard, Amandine Le Bourgeois, Michel Chérel, Camille Debord, Yannick Le Bris, Olivier Theisen, et al.

\section{To cite this version:}

Pierre Peterlin, Joëlle Gaschet, Thierry Guillaume, Alice Garnier, Marion Eveillard, et al.. Fms-like Tyrosine Kinase 3 Ligand Concentration Kinetic Profile during Induction Is Strongly Predictive of Survivals in AML: Results of the FLAM/Flal Study. Blood, 2018, 132 (Supplement 1), pp.1484-1484. 10.1182/blood-2018-99-111694 . inserm-02341909

\section{HAL Id: inserm-02341909 https://www.hal.inserm.fr/inserm-02341909}

Submitted on 31 Oct 2019

HAL is a multi-disciplinary open access archive for the deposit and dissemination of scientific research documents, whether they are published or not. The documents may come from teaching and research institutions in France or abroad, or from public or private research centers.
L'archive ouverte pluridisciplinaire HAL, est destinée au dépôt et à la diffusion de documents scientifiques de niveau recherche, publiés ou non, émanant des établissements d'enseignement et de recherche français ou étrangers, des laboratoires publics ou privés. 
Fms-like Tyrosine Kinase 3 Ligand Concentration Kinetic Profile during Induction Is Strongly Predictive of Survivals in AML: Results of the FLAM/Flal Study

Pierre Peterlin, Joelle Gaschet, Thierry Guillaume, Alice Garnier, Marion Eveillard, Amandine Le Bourgeois, Michel Cherel, Camille Debord, Yannick LE Bris, Olivier Theisen, Beatrice Mahe, Viviane Dubruille, Soraya Wuilleme, Catherine Godon, Cyrille Touzeau, Thomas Gastinne, Nicolas Blin, Anne Lok, Antoine Bonnet, Steven LE Gouill, Philippe Moreau, Marie C Bene and Patrice Chevallier

Blood 2018 132:1484; doi: https://doi.org/10.1182/blood-2018-99-111694

Published By American Society of Hematology

Print ISSN 0006-4971

Online ISSN 1528-0020

Published online November 21, 2018.

Copyright \& Usage (C) 2018 by The American Society of Hematology

Contributors

Pierre Peterlin, 1Clinical Hematology, Nantes University Hospital, Nantes, France 2CRCINA, INSERM, CNRS, Université d'Angers, Université de Nantes, Nantes, France

Joelle Gaschet [PhD], 2CRCINA, INSERM, CNRS, Université d'Angers, Université de Nantes, Nantes, France

Thierry Guillaume [MD PhD], 1Clinical Hematology, Nantes University Hospital, Nantes, France 2CRCINA, INSERM, CNRS, Université d'Angers, Université de Nantes, Nantes, France

Alice Garnier [MD], 1Clinical Hematology, Nantes University Hospital, Nantes, France

Marion Eveillard [PharmD, PhD], 2CRCINA, INSERM, CNRS, Université d'Angers, Université de Nantes, Nantes, France 3Hematology Biology, Nantes University Hospital, Nantes, France

Amandine Le Bourgeois [MD], 1Clinical Hematology, Nantes University Hospital, Nantes, France

Michel Cherel [MD PhD], 2CRCINA, INSERM, CNRS, Université d'Angers, Université de Nantes, Nantes, France 4Nuclear Medicine Unit, ICO Cancer Center Gauducheau, Saint Herblain, France

Camille Debord [PharmD], 3Hematology Biology, Nantes University Hospital, Nantes, France Yannick LE Bris [MDPharmD], 5Hematology Biology, CHU Nantes, Nantes, France 2CRCINA, INSERM, CNRS, Université d'Angers, Université de Nantes, Nantes, France

Olivier Theisen [MD], 3Hematology Biology, Nantes University Hospital, Nantes, France

Beatrice Mahe [MD], 1Clinical Hematology, Nantes University Hospital, Nantes, France

Viviane Dubruille [MD], 1Clinical Hematology, Nantes University Hospital, Nantes, France

Soraya Wuilleme [PharmD], 3Hematology Biology, Nantes University Hospital, Nantes, France

Catherine Godon [MD], 3Hematology Biology, Nantes University Hospital, Nantes, France

Cyrille Touzeau [MD PhD], 6Hematology Clinic, Nantes University Hospital, Nantes, France 
Thomas Gastinne [MD], 1Clinical Hematology, Nantes University Hospital, Nantes, France Nicolas Blin [MD], 1Clinical Hematology, Nantes University Hospital, Nantes, France Anne Lok [MD], 1Clinical Hematology, Nantes University Hospital, Nantes, France Antoine Bonnet [MD], 1Clinical Hematology, Nantes University Hospital, Nantes, France Steven LE Gouill [MD PhD], 1Clinical Hematology, Nantes University Hospital, Nantes, France 2CRCINA, INSERM, CNRS, Université d'Angers, Université de Nantes, Nantes, France Philippe Moreau, 7University Hospital Hôtel-Dieu, Nantes, France 8CRCINA, INSERM, CNRS, Angers University and Nantes University, Nantes, France

Marie C Bene [PharmSci, PhD], 2CRCINA, INSERM, CNRS, Université d'Angers, Université de Nantes, Nantes, France 3Hematology Biology, Nantes University Hospital, Nantes, France

Patrice Chevallier [MD], 2CRCINA, INSERM, CNRS, Université d'Angers, Université de Nantes, Nantes, France 1Clinical Hematology, Nantes University Hospital, Nantes, France 


\section{Abstract}

The cytokine Fms-like tyrosine kinase 3 ligand (FL) is a key regulator of hematopoiesis. In a previous Phase 1 study testing a radioimmunotherapy regimen for relapsed/refractory acute lymphoblastic leukemia ( $A L L)$, responders showed increased soluble $F L$ serum concentration (sFLc) after salvage regimen (Chevallier, Lancet Haematol., 2015).

This prospective monocentric study (ClinicalTrials.gov NCT02693899) aimed to assess the impact of SFLc in ALL and acute myeloid leukemia (AML) patients treated according to standard-of-care intensive first-line chemotherapy regimens. Serum samples were collected on days $1,8,15,22$ of induction, at days 1, 8, 15 of each intensive consolidation or day 1 of each non intensive consolidation when appropriate, frozen-stored then tested by ELISA (DY308, R\&D Systems, Minneapolis, MN). The following outcomes were considered to assess the impact of sFLc: refractory status after induction ( $\geq 5 \%$ bone marrow blasts or persistent aplasia $>45$ days), morphologic, immunophenotypic, cytogenetic or molecular relapses, eventfree (EFS) and overall survival (OS). All patients provided informed consent.

Between May 2016 and January 2018, 80 patients were included. Data were ultimately available for $16 \mathrm{ALL}$ and $62 \mathrm{AML}$ patients. A total of 579 samples were assayed. Analysis of the results disclosed 3 sFLc kinetic profiles during induction i) sustained increase from days 1 to 22 (FLI group), ii) increase from days 1 to 15, then decrease at day 22 (FLD group) and iii) stagnation of low levels all along ( $<1000 \mathrm{pg} / \mathrm{mL}$ from days 1 to 22 , FLL group).

The 16 evaluable ALL patients were classified as FLI ( $n=2), \operatorname{FLD}(n=7)$ and FLL $(n=7)$. All reached a cytologic complete remission after induction and only 2 relapses have been documented so far in this group. No impact of sFLc kinetic profile was seen in this context.

Conversely, a significant impact of sFLc during induction (but not during consolidation) was observed in AML patients. The median age in this group was 59 years old (range: $29-71,<60$ years $n=33$ ). The median follow-up for alive patients was 541 days (range: 154-787). sFLc levels were assayed in 244 samples. Twenty-six patients were classified as FLI (42\%), 22 as FLD (35\%) and 14 as FLL (23\%). Median sFLc at days 1, 8, 15, 22 were as follows for the three groups: FLI: $2,724,3673,5753$ pg/mL; FLD: 6, 1229, 6019, $684 \mathrm{pg} / \mathrm{mL}$; and FLL: 0, 60, 124, $81 \mathrm{pg} / \mathrm{mL}$. There was no significant difference between the 3 groups regarding age, ELN 2010 risk-stratification (ELNrs), OMS classification, WBC and bone marrow blasts percentages at diagnosis.

When comparing the 3 sFLc groups, almost all refractory patients $(n=6)$ were found in the FLL group ( $n=5, F L D n=1, F L I n=0, p=0.0007$ ). Three cytologic relapses occurred in the FLI group, 7 in the FLD group (cytologic $n=4$, molecular $n=2$, immunophenotypic $n=1$ ) and 7 in the FLL group (cytologic $n=4$, molecular $n=2$, immunophenotypic $n=1$ ). There were more relapses in the FLL group ( $n=7 / 9$ [78\%] vs FLD $n=7 / 21$ [33\%] vs FLI $n=3 / 26$ [11.5\%], $p=0.0009$ ).

In univariate analysis, 2-year EFS and OS were significantly better for the FLI group (79.1+-8 vs FLD $54.9 \%+-11$ vs FLL $11.4 \%+-10, p<0.001$; and $80.4 \%+-8$ vs FLD $58.6 \%+-11$ vs FLL $18.6 \%+-10$, $\mathrm{p}=0.09$, respectively). There was a trend for the association of 2-year EFS (but not OS) with ELNrs (favorable:70.9\%+-11, vs Int-1+Int-2:57.1\%+-10 vs adverse $33 \%+-13, p=0.06$ ). Stratification of the patients according to the median sFLc level at day $+15(2952 \mathrm{pg} / \mathrm{mL})$ also 
showed significantly different 2 year EFS at $38.2 \%+-9$ for low levels vs $71.8 \%+-8$ for high levels $(p=0.02)$. The same was true for day +22 median $s F L c$ level $(1390 \mathrm{pg} / \mathrm{mL})$ at $38.9 \%+-9 \mathrm{vs}$ $73.6 \%+-8(p=0.02)$. Age had no impact on EFS nor OS.

In multivariate analysis considering age, ELNrs, sFLc at days 15 and 22 levels, and sFLc kinetic profile during induction, the latter remained the most powerful factor independently associated with EFS (HR: 3.62 ; 95\%Cl: 1,65-7,94, $p=0,001$; ELNrs: HR: 1.74; 95\%Cl: 0,98-3.10, $p=0.05$; sFLc at day $+15 p=0,37$; sFLc at day $+22, p=0.24$, age $p=N S)$. sFLc kinetic profile was the sole factor that was also independently associated with OS (HR: $2.60 ; 95 \% \mathrm{Cl}: 1.12-6,07$, $\mathrm{p}=0.02$ ).

In conclusion, sFLc kinetic profile during induction appears to be a new powerful early prognostic parameter in AML patients. These results need to be validated on a larger cohort of patients and the mechanism by which induction sFLc levels may impact AML outcome remains to be elucidated.

Figure 1: EFS and OS according to three $s$ FLc kinetic profiles

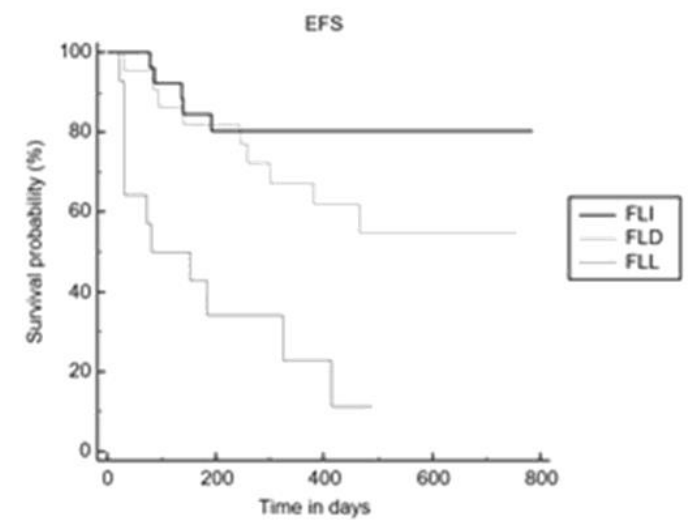

$\mathrm{P}<0.0001$

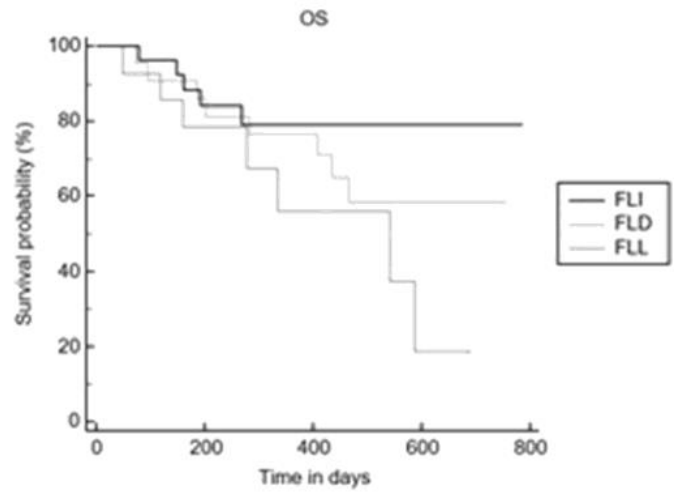

$P=0.09$

Disclosures Gastinne:

Millennium/Takeda: Honoraria. Moreau: Abbvie: Honoraria, Membership on an entity's Board of Directors or advisory committees; Janssen: Honoraria, Membership on an entity's Board of Directors or advisory committees; Celgene: Honoraria, Membership on an entity's Board of Directors or advisory committees; Amgen: Honoraria, Membership on an entity's Board of Directors or advisory committees; Takeda: Honoraria, Membership on an entity's Board of Directors or advisory committees. 